\title{
Dinuclear Bis(1,2-diaryl-1,2-ethylenedithiolato)iron
}

\section{Complexes: $\left[\mathrm{Fe}_{2}^{\mathrm{III}}(\mathrm{L})_{4}\right]^{\mathrm{n}}(\mathrm{n}=2-, 1-, 0,1+)$}

Apurba Patra $^{a)}$, Eckhard Bill ${ }^{a)}$, Thomas Weyhermüller ${ }^{a)}$, Keira Stobie ${ }^{b)}$, Zoe Bell ${ }^{b)}$, Michael D. Ward ${ }^{(c)}$, Jon A. McCleverty ${ }^{b}$, Karl Wieghardt ${ }^{*}$

a) Max-Planck-Institut für Bioanorganische Chemie, Stiftstrasse 34-36, D-45470 Mülheim an der Ruhr, Germany

b) School of Chemistry, University of Bristol, Bristol BS8 1TS, UK

c) Department of Chemistry, University Sheffield, Sheffield S3 7HF, UK

E-mail: wieghardt@mpi-muelheim.mpg.de

RECEIVED DATE (to be automatically inserted after your manuscript is accepted if required according to the journal that you are submitting your paper to)

\section{Supporting Information:}




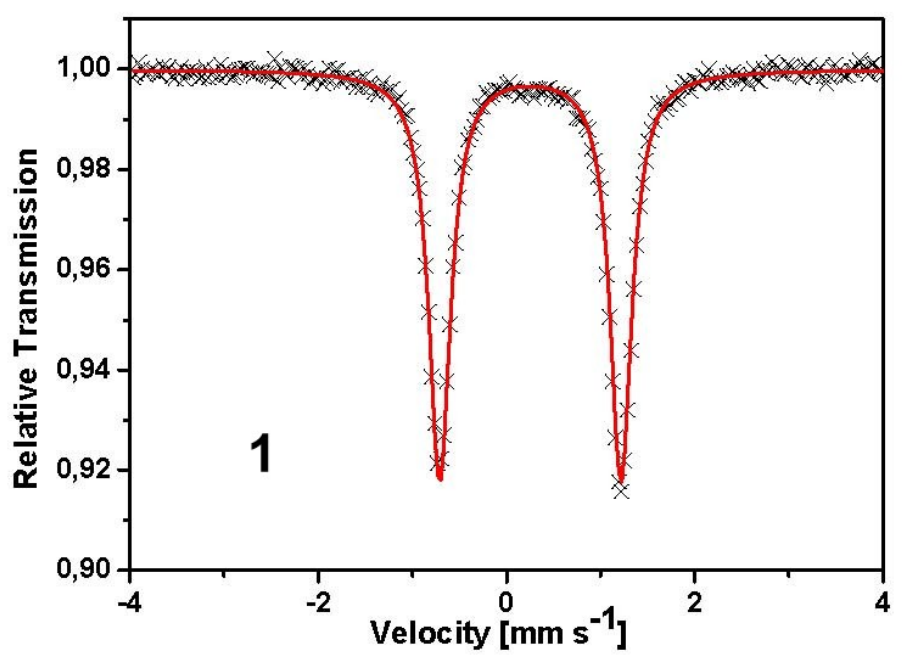

Figure S1. Zero-field Mössbauer spectrum of a solid sample of $\mathbf{1}$ at $80 \mathrm{~K}$. Isomer shift $\delta=0.25 \mathrm{~mm} \mathrm{~s}^{-1}$, quadrupole splitting $\Delta \mathrm{E}_{\mathrm{Q}}=1.93 \mathrm{~mm} \mathrm{~s}^{-1}$, width at half hight $0.28 \mathrm{~mm} \mathrm{~s}^{-1}$.

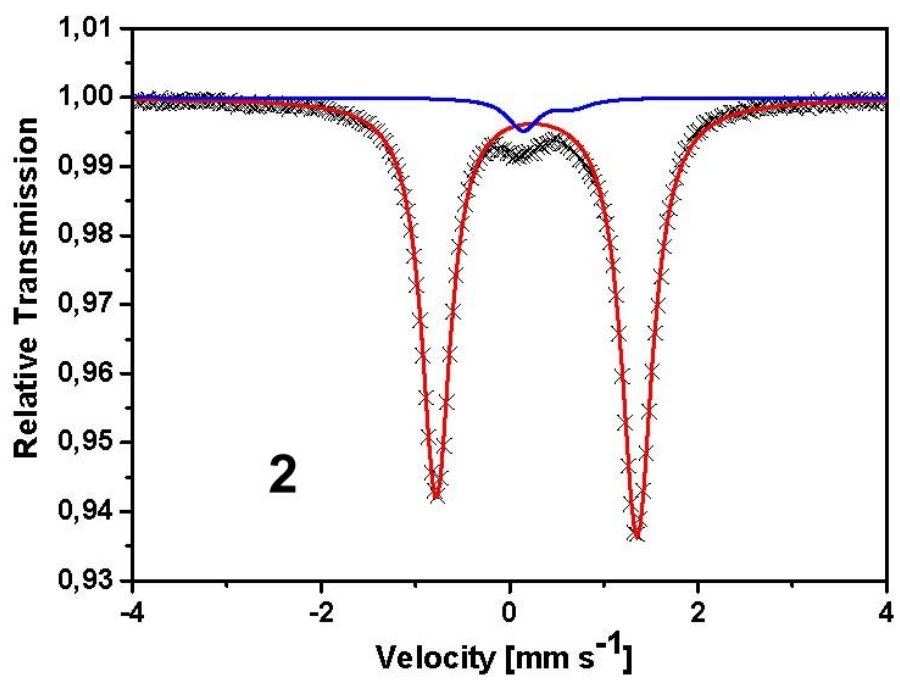

Figure S2. Zero-field Mössbauer spectrum of a solid sample of 2 at $80 \mathrm{~K}$. Subspectrum a (red) $96 \%: \delta=0.29 \mathrm{~mm} \mathrm{~s}^{-1}, \Delta \mathrm{E}_{\mathrm{Q}}=2.13 \mathrm{~mm} \mathrm{~s}^{-1}$; Subspectrum b (blue) $4 \%: \delta=0.42 \mathrm{~mm} \mathrm{~s}^{-1}, \Delta \mathrm{E}_{\mathrm{Q}}=0.55 \mathrm{~mm} \mathrm{~s}^{-1}$. 


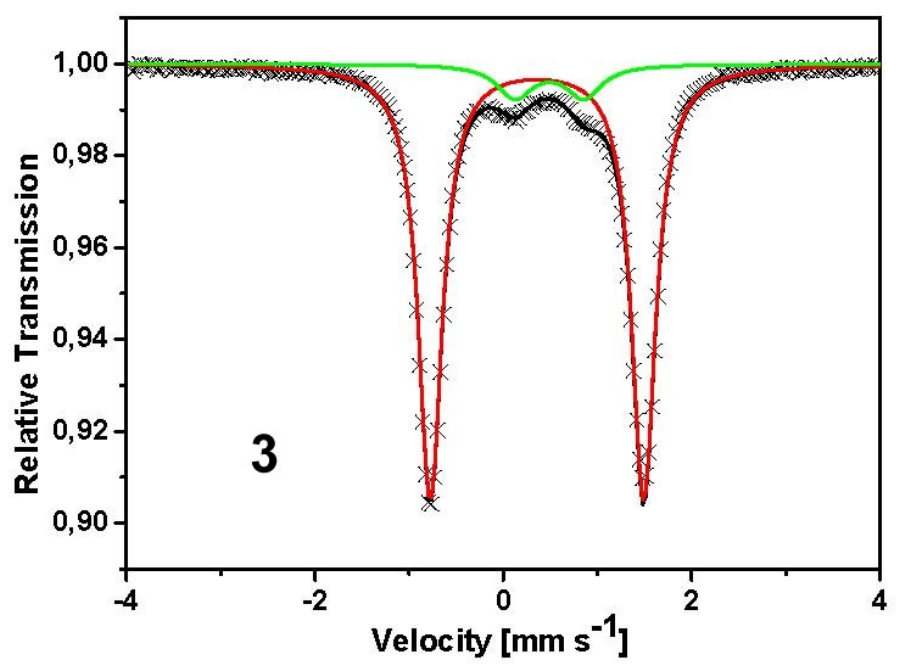

Figure S3. Zero-field Mössbauer spectrum of a solid sample of $\mathbf{3}$ at $80 \mathrm{~K}$. Subspectrum a (red) $91 \%: \delta=0.36 \mathrm{~mm} \mathrm{~s}^{-1}, \Delta \mathrm{E}_{\mathrm{Q}}=2.26 \mathrm{~mm} \mathrm{~s}^{-1}$; Subspectrum b (green) $9 \%: \delta=0.49 \mathrm{~mm} \mathrm{~s}^{-1}, \Delta \mathrm{E}_{\mathrm{Q}}=0.73 \mathrm{~mm} \mathrm{~s}^{-1}$. 